\title{
M-positive semi-definiteness and M-positive definiteness of fourth-order partially symmetric Cauchy tensors
}

\author{
Haitao Che ${ }^{1 *}$, Haibin Chen ${ }^{2}$ and Yiju Wang ${ }^{2}$
}

"Correspondence:

haitaoche@163.com

${ }^{1}$ School of Mathematics and

Information Science, Weifang University, Weifang, China

Full list of author information is

available at the end of the article

\begin{abstract}
Inspired by symmetric Cauchy tensors, we define fourth-order partially symmetric Cauchy tensors with their generating vectors. In this article, we focus on the necessary and sufficient conditions for the M-positive semi-definiteness and M-positive definiteness of fourth-order Cauchy tensors. Moreover, the necessary and sufficient conditions of the strong ellipticity conditions for fourth-order Cauchy tensors are obtained. Furthermore, fourth-order Cauchy tensors are M-positive semi-definite if and only if the homogeneous polynomial for fourth-order Cauchy tensors is monotonically increasing. Several M-eigenvalue inclusion theorems and spectral properties of fourth-order Cauchy tensors are discussed. A power method is proposed to compute the smallest and the largest M-eigenvalues of fourth-order Cauchy tensors. The given numerical experiments show the effectiveness of the proposed method.
\end{abstract}

MSC: 90C30; 15A06; 74B99; 15A18; 15A69

Keywords: Cauchy tensor; M-positive semi-definite; M-positive definite; Spectral property; Homogeneous polynomial; M-eigenvalue; Power method

\section{Introduction}

Let $\mathbb{R}^{n}$ be an $n$-dimensional real Euclidean space and denote the set consisting of all natural numbers by $N$. Suppose that $m$ and $n$ are positive natural numbers and write $[n]=\{1,2, \ldots, n\}$. The nonlinear elastic materials analysis and entanglement studies in quantum physics can be formulated as the following optimization problem:

$$
\left\{\begin{array}{l}
\max f(\mathbf{x}, \mathbf{y})=\sum_{i, k \in[m]} \sum_{j, l \in[n]} c_{i j k l} x_{i} y_{j} x_{k} y_{l}, \\
\text { s.t. } \mathbf{x}^{T} \mathbf{x}=1, \mathbf{y}^{T} \mathbf{y}=1, \\
\quad \mathbf{x} \in \mathbb{R}^{m}, \mathbf{y} \in \mathbb{R}^{n},
\end{array}\right.
$$

where the coefficients $c_{i j k l}$ satisfy the following symmetric property:

$$
c_{i j k l}=c_{k j i l}=c_{i l k j}=c_{k l i j}, \quad i, k \in[m], j, l \in[n] .
$$

(c) The Author(s) 2019. This article is distributed under the terms of the Creative Commons Attribution 4.0 International License (http://creativecommons.org/licenses/by/4.0/), which permits unrestricted use, distribution, and reproduction in any medium, provided you give appropriate credit to the original author(s) and the source, provide a link to the Creative Commons license, and indicate if changes were made. 
Then, the tensor $\mathcal{C}=\left(c_{i j k l}\right)$ is called to be a partially symmetric fourth-order tensor. In the nonlinear elastic materials analysis, one approach is to consider an elastic material in terms of a fourth-order three-dimensional elastic module tensor that satisfies the partially symmetric property [1]. Thus, the partially symmetric property of tensor is becoming to be an interesting subject. Inspired by Cauchy matrix and Cauchy tensor, we will construct a new kind of tensor which satisfies the partially symmetric property.

An $m \times n$ Cauchy matrix assigned to $m+n$ parameters $x_{1}, x_{2}, \ldots, x_{m}, y_{1}, \ldots, y_{n}$ was introduced by [2] as follows:

$$
C=\left[\frac{1}{x_{i}+y_{j}}\right], \quad i \in[m], j \in[n] .
$$

The Cauchy matrix has played an important role in algorithm designing [3-5]. If $x_{i}=y_{i}$ and $m=n$ in (1.2), then it reduces to a real symmetric Cauchy matrix. Motivated by symmetric Cauchy matrices, Chen and Qi [6] proposed the definition of Cauchy tensors.

Definition 1 Let a vector $\mathbf{c}=\left(c_{1}, c_{2}, \ldots, c_{n}\right) \in \mathbb{R}^{n}$. Suppose that a real tensor $\mathcal{C}=\left(c_{i_{1} i_{2} \cdots i_{m}}\right)$ is defined by

$$
c_{i_{1} i_{2} \cdots i_{m}}=\frac{1}{c_{i_{1}}+c_{i_{2}}+\cdots+c_{i_{m}}}, \quad i_{j} \in[n], j \in[m] .
$$

Then $\mathcal{C}$ is called an order $m$ dimension $n$ symmetric Cauchy tensor and the vector $\mathbf{c} \in \mathbb{R}^{n}$ is called the generating vector of $\mathcal{C}$.

Following the ideas of Cauchy matrix [2] and Cauchy tensor [6], we present the definition of fourth-order Cauchy tensors.

Definition 2 Let a vector $\mathbf{a}=\left(a_{1}, a_{2}, \ldots, a_{m}\right) \in \mathbb{R}^{m}$ and a vector $\mathbf{b}=\left(b_{1}, b_{2}, \ldots, b_{n}\right) \in \mathbb{R}^{n}$. Suppose that a real tensor $\mathcal{C}=\left(c_{i j k l}\right)$ is defined by

$$
c_{i j k l}=\frac{1}{a_{i}+b_{j}+a_{k}+b_{l}}, \quad i, k \in[m], j, l \in[n] .
$$

Then we claim that $\mathcal{C}$ is a fourth-order Cauchy tensor and the vectors $\mathbf{a} \in \mathbb{R}^{m}, \mathbf{b} \in \mathbb{R}^{n}$ are called generating vectors of $\mathcal{C}$.

Obviously, the fourth-order Cauchy tensor has the following partially symmetric property:

$$
c_{i j k l}=c_{k j i l}=c_{i l k j}=c_{k l i j}=\frac{1}{a_{i}+b_{j}+a_{k}+b_{l}}, \quad i, k \in[m], j, l \in[n] .
$$

Furthermore, if $\mathbf{a}=\mathbf{b}$ and $m=n$, then the fourth-order partially symmetric Cauchy tensor reduces to the fourth-order symmetric Cauchy tensor. In Definition 2, we point out that, for the generating vectors $a$ and $b$, it should satisfy

$$
a_{i}+b_{j}+a_{k}+b_{l} \neq 0, \quad i, k \in[m], j, l \in[n] .
$$


In this paper, we always consider the fourth-order real partially symmetric Cauchy tensors. Hence, they can be called fourth-order Cauchy tensors for simplicity.

Recently, a lot of researchers have focused on structured tensors [6-28] such as Mtensors, Hankel tensors, Hilbert tensors, Cauchy tensors, completely positive tensors, B-tensors, and P-tensors. These papers not only gave some results on positive semidefiniteness property and spectral theory of structured tensors, but also revealed some important applications in data fitting and stochastic process [10, 29].

In this article, we focus on the M-positive semi-definiteness and M-positive definiteness conditions for fourth-order Cauchy tensors. Several spectral properties of M-positive semi-definite fourth-order Cauchy tensors are discussed. A power method is proposed to compute the smallest and the largest M-eigenvalues of fourth-order Cauchy tensors. In Sect. 2, the necessary and sufficient conditions for M-positive semi-definiteness and Mpositive definiteness of fourth-order Cauchy tensors are obtained. Moreover, the necessary and sufficient conditions of the strong ellipticity condition for fourth-order Cauchy tensors are obtained. Furthermore, fourth-order Cauchy tensors are M-positive semidefinite if and only if the homogeneous polynomial of fourth-order Cauchy tensors is monotonically increasing in the nonnegative orthant of $\mathbb{R}^{m} \times \mathbb{R}^{n}$, and the homogeneous polynomial is strictly monotone increasing when fourth-order Cauchy tensors are Mpositive definite. In Sect. 3, several spectral inequalities are presented on the M-eigenvalue of fourth-order Cauchy tensors. We introduce a power method to compute the smallest and the largest M-eigenvalues of fourth-order Cauchy tensors, and numerical experiments show the effectiveness of the proposed method in Sect. 4.

At the end of the introduction, we make some notations that will be applied to the sequel. Denote vectors by lowercase boldface letters, i.e., $\mathbf{x}, \mathbf{y}, \ldots$, and tensors are written to calligraphic capitals such as $\mathcal{A}, \mathcal{T}, \ldots$ For $\mathbf{x}=\left(x_{1}, x_{2}, \ldots, x_{n}\right), \mathbf{y}=\left(y_{1}, y_{2}, \ldots, y_{n}\right), \mathbf{x} \geq \mathbf{y}$ $(\mathbf{x} \leq \mathbf{y})$ means $x_{i} \geq y_{i}\left(x_{i} \leq y_{i}\right)$ for all $i \in[n]$.

\section{M-positive semi-definiteness and M-positive definiteness of fourth-order Cauchy tensors}

Let

$$
f(\mathbf{x}, \mathbf{y})=\mathcal{C} \mathbf{x y x y}=\sum_{i, k \in[m], j, l \in[n]} c_{i j k l} x_{i} y_{j} x_{k} y_{l} .
$$

The tensor $\mathcal{C}$ is called M-positive semi-definite if $f(\mathbf{x}, \mathbf{y}) \geq 0$ for any vectors $\mathbf{x} \in \mathbb{R}^{m}, \mathbf{y} \in \mathbb{R}^{n}$. The tensor $\mathcal{C}$ is called M-positive definite if $f(\mathbf{x}, \mathbf{y})>0$ for any vectors $\mathbf{x} \in \mathbb{R}^{m}, \mathbf{x} \neq \mathbf{0}, \mathbf{y} \in \mathbb{R}^{n}$, $\mathbf{y} \neq \mathbf{0}$. Similarly, the tensor $\mathcal{C}$ is M-negative semi-definite (M-negative definite) if $f(\mathbf{x}, \mathbf{y}) \leq 0$ for any vectors $\mathbf{x} \in \mathbb{R}^{m}, \mathbf{y} \in \mathbb{R}^{n}\left(f(\mathbf{x}, \mathbf{y})<0\right.$ for any vectors $\left.\mathbf{x} \in \mathbb{R}^{m}, \mathbf{x} \neq \mathbf{0}, \mathbf{y} \in \mathbb{R}^{n}, \mathbf{y} \neq \mathbf{0}\right)$.

Now, we will show some necessary and sufficient conditions for fourth-order Cauchy tensors to be M-positive semi-definite.

Theorem 2.1 Let the vectors $\mathbf{a} \in \mathbb{R}^{m}, \mathbf{b} \in \mathbb{R}^{n}$ be generating vectors of the fourth-order Cauchy tensor $\mathcal{C}$. Then the tensor $\mathcal{C}$ is $M$-positive semi-definite if and only if $a_{i}+b_{j}>0$ for all $i \in[m], j \in[n]$. 
Proof From the fact that the Cauchy tensor $\mathcal{C}$ is M-positive semi-definite, taking $\mathbf{x}=\mathbf{e}_{i} \in$ $\mathbb{R}^{m}$ and $\mathbf{y}=\mathbf{e}_{j} \in \mathbb{R}^{n}$, we have

$$
\begin{aligned}
f\left(\mathbf{e}_{i}, \mathbf{e}_{j}\right) & =\mathcal{C} \mathbf{e}_{i} \mathbf{e}_{j} \mathbf{e}_{i} \mathbf{e}_{j} \\
& =\frac{1}{2\left(a_{i}+b_{j}\right)} \geq 0, \quad i \in[m], j \in[n],
\end{aligned}
$$

where $\mathbf{e}_{i}$ and $\mathbf{e}_{j}$ are the $i$ th and $j$ th coordinate vectors, respectively. Thus, we have $a_{i}+b_{j}>0$ for all $i \in[m], j \in[n]$.

On the other hand, suppose that $a_{i}+b_{j}>0$ for all $i \in[m], j \in[n]$. For any $\mathbf{x} \in \mathbb{R}^{m}, \mathbf{y} \in \mathbb{R}^{n}$, one has

$$
\begin{aligned}
f(\mathbf{x}, \mathbf{y}) & =\mathcal{C} \mathbf{x y x y}=\sum_{i, k \in[m], j, l \in[n]} c_{i j k l} x_{i} y_{j} x_{k} y_{l} \\
& =\sum_{i, k \in[m], j, l \in[n]} \frac{x_{i} y_{j} x_{k} y_{l}}{a_{i}+b_{j}+a_{k}+b_{l}} \\
& =\sum_{i, k \in[m], j, l \in[n]} \int_{0}^{1} t^{a_{i}+b_{j}+a_{k}+b_{l}-1} x_{i} y_{j} x_{k} y_{l} d t \\
& =\int_{0}^{1}\left(\sum_{i \in[m]} t^{a_{i}-\frac{1}{4}} x_{i}\right)^{2}\left(\sum_{j \in[n]} t^{b_{j}-\frac{1}{4}} y_{j}\right)^{2} d t \\
& \geq 0,
\end{aligned}
$$

which means that the tensor $\mathcal{C}$ is M-positive semi-definite and the conclusion follows.

From Theorem 2.1, we deduce the following corollary directly.

Corollary 2.1 Assume that the fourth-order Cauchy tensor $\mathcal{C}$ and its generating vectors $\mathbf{a} \in \mathbb{R}^{m}, \mathbf{b} \in \mathbb{R}^{n}$ are defined in Theorem 2.1. Then the tensor $\mathcal{C}$ is $M$-negative semi-definite if and only if $a_{i}+b_{j}<0$ for all $i \in[m], j \in[n]$.

Corollary 2.2 Assume that the fourth-order Cauchy tensor $\mathcal{C}$ and its generating vectors $\mathbf{a} \in \mathbb{R}^{m}, \mathbf{b} \in \mathbb{R}^{n}$ are defined in Theorem 2.1. Then the tensor $\mathcal{C}$ is not $M$-positive semi-definite if and only if there exist at least $i \in[m], j \in[n], a_{i}+b_{j}<0$ holds.

Next, we will reveal some necessary and sufficient conditions for fourth-order Cauchy tensors to be M-positive definite.

Theorem 2.2 Assume that the vectors $\mathbf{a} \in \mathbb{R}^{m}, \mathbf{b} \in \mathbb{R}^{n}$ are generating vectors of the fourthorder Cauchy tensor $\mathcal{C}$. For all $i \in[m], j \in[n]$, if $a_{i}+b_{j}>0$ and the elements of generating vectors $\mathbf{a}, \mathbf{b}$ are mutually distinct, respectively, then the tensor $\mathcal{C}$ is $M$-positive definite.

Proof It follows from Theorem 2.1 that the tensor $\mathcal{C}$ is M-positive semi-definite. We prove by contradiction that the tensor $\mathcal{C}$ is $\mathrm{M}$-positive definite. Assume that there exists nonzero vectors $\mathbf{x} \in \mathbb{R}^{m}, \mathbf{y} \in \mathbb{R}^{n}$ such that

$$
f(\mathbf{x}, \mathbf{y})=\mathcal{C} \mathbf{x y x y}=0 .
$$


Following the proof of Theorem 2.1, we deduce

$$
\int_{0}^{1}\left(\sum_{i \in[m]} t^{a_{i}-\frac{1}{4}} x_{i}\right)^{2}\left(\sum_{j \in[n]} t^{b_{j}-\frac{1}{4}} y_{j}\right)^{2} d t=0
$$

which implies

$$
\sum_{i \in[m]} t^{a_{i}-\frac{1}{4}} x_{i} \equiv 0, \quad t \in(0,1]
$$

or

$$
\sum_{j \in[m]} t^{b_{j}-\frac{1}{4}} y_{j} \equiv 0, \quad t \in(0,1] .
$$

Without loss of generality, we can assume

$$
\sum_{i \in[m]} t^{a_{i}-\frac{1}{4}} x_{i} \equiv 0, \quad t \in(0,1] .
$$

Then

$$
x_{1}+t^{a_{2}-a_{1}} x_{2}+\cdots+t^{a_{m}-a_{1}} x_{m} \equiv 0, \quad t \in(0,1] .
$$

By the continuity and the fact that $a_{1}, a_{2}, \ldots, a_{m}$ are mutually distinct, it yields that

$$
x_{1}=0 \text {, }
$$

and

$$
x_{2}+t^{a_{3}-a_{2}} x_{3}+\cdots+t^{a_{m}-a_{2}} x_{m} \equiv 0, \quad t \in(0,1] .
$$

Applying the same argument, one has

$$
x_{1}=x_{2}=\cdots=x_{m}=0 \text {, }
$$

which is a contradiction with $\mathbf{x} \neq \mathbf{0}$. So, we conclude that the tensor $\mathcal{C}$ is $\mathrm{M}$-positive definite.

Moreover, the following conclusion shows that the conditions in Theorem 2.2 are necessary and sufficient conditions.

Theorem 2.3 Assume that the fourth-order Cauchy tensor $\mathcal{C}$ and its generating vectors a, $\mathbf{b}$ are defined in Theorem 2.2. The tensor $\mathcal{C}$ is $M$-positive definite if and only if $a_{i}+b_{j}>0$ for all $i \in[m], j \in[n]$, and the elements of generating vectors $\mathbf{a}, \mathbf{b}$ are mutually distinct, respectively. 
Proof From Theorem 2.2, if $a_{i}+b_{j}>0$ for all $i \in[m], j \in[n]$ and the elements of generating vectors $\mathbf{a}, \mathbf{b}$ are mutually distinct, respectively, then we can obtain that the tensor $\mathcal{C}$ is $\mathrm{M}$ positive definite. Next, suppose that the tensor $\mathcal{C}$ is M-positive definite, we will reveal that $a_{i}+b_{j}>0$ for all $i \in[m], j \in[n]$ and the elements of generating vectors $\mathbf{a}, \mathbf{b}$ are mutually distinct, respectively. Indeed, from Theorem 2.1, we know that the tensor $\mathcal{C}$ is $\mathrm{M}$-positive semi-definite; therefore, $a_{i}+b_{j}>0$ for all $i \in[m], j \in[n]$. By contradiction, we can assume that two elements of the vector $\mathbf{a}$ are equal. Without loss of generality, suppose that $a_{1}=$ $a_{2}=\bar{a}$. Let $\mathbf{x}=(1,-1,0, \ldots, 0) \in \mathbb{R}^{m}$ and $\mathbf{y}=(1,0,0, \ldots, 0) \in \mathbb{R}^{n}$. Thus, we have

$$
\begin{aligned}
\mathcal{C} \text { xyxy } & =\sum_{i, k \in[m], j, l \in[n]} c_{i j k l} x_{i} y_{j} x_{k} y_{l} \\
& =\sum_{i, k \in[m], j, l \in[n]} \frac{x_{i} y_{j} x_{k} y_{l}}{a_{i}+b_{j}+a_{k}+b_{l}} \\
& =\frac{1}{2\left(\bar{a}+b_{1}\right)} \sum_{i, k \in[2]} x_{i} x_{k} \\
& =\frac{1}{2\left(\bar{a}+b_{1}\right)}[1 \cdot 1+(-1) \cdot 1+1 \cdot(-1)+(-1) \cdot(-1)] \\
& =0,
\end{aligned}
$$

which contradicts the assumption that the tensor $\mathcal{C}$ is $\mathrm{M}$-positive definite and the proof is completed.

In what follows, we will give the definition of the monotonicity of a homogeneous polynomial with respect to fourth-order Cauchy tensors.

For any $\mathbf{x}, \overline{\mathbf{x}} \in \mathbb{R}^{m}$ and $\mathbf{y}, \overline{\mathbf{y}} \in \mathbb{R}^{n}$, if $f(\mathbf{x}, \mathbf{y}) \geq f(\overline{\mathbf{x}}, \overline{\mathbf{y}})$ when $\mathbf{x} \geq \overline{\mathbf{x}}$ and $\mathbf{y} \geq \overline{\mathbf{y}},(\mathbf{x} \leq \overline{\mathbf{x}}$ and $\mathbf{y} \leq$ $\overline{\mathbf{y}})$, then $f(\mathbf{x}, \mathbf{y})$ is called monotonically increasing (monotonically decreasing respectively). If $f(\mathbf{x}, \mathbf{y})>f(\overline{\mathbf{x}}, \overline{\mathbf{y}})$ when $\mathbf{x} \geq \overline{\mathbf{x}}, \mathbf{x} \neq \overline{\mathbf{x}}$ and $\mathbf{y} \geq \overline{\mathbf{y}}, \mathbf{y} \neq \overline{\mathbf{y}}(\mathbf{x} \leq \overline{\mathbf{x}}, \mathbf{x} \neq \overline{\mathbf{x}}$ and $\mathbf{y} \leq \overline{\mathbf{y}}, \mathbf{y} \neq \overline{\mathbf{y}})$, then $f(\mathbf{x}, \mathbf{y})$ is called strictly monotone increasing (strictly monotone decreasing respectively).

The following conclusions reveal the relationships between M-positive semidefiniteness of fourth-order Cauchy tensor and the monotonicity of a homogeneous polynomial with respect to the proposed Cauchy tensor.

Theorem 2.4 Let $\mathcal{C}$ be a fourth-order Cauchy tensor with generating vectors $\mathbf{a} \in \mathbb{R}^{m}$ and $\mathbf{b} \in \mathbb{R}^{n}$. Then the tensor $\mathcal{C}$ is $M$-positive semi-definite if and only if the homogeneous polynomial $f(\mathbf{x}, \mathbf{y})$ is monotonically increasing in $\mathbb{R}_{+}^{m} \times \mathbb{R}_{+}^{n}$.

Proof For sufficiency, let $\mathbf{x}=\mathbf{e}_{i} \in \mathbb{R}_{+}^{m}, \overline{\mathbf{x}}=\mathbf{0} \in \mathbb{R}_{+}^{m}$ and $\mathbf{y}=\mathbf{e}_{j} \in \mathbb{R}_{+}^{n}, \overline{\mathbf{y}}=\mathbf{0} \in \mathbb{R}_{+}^{n}$, one has

$$
\frac{1}{2\left(a_{i}+b_{j}\right)}=\mathcal{C} \mathbf{x y x y}=f(\mathbf{x}, \mathbf{y}) \geq f(\overline{\mathbf{x}}, \overline{\mathbf{y}})=\mathcal{C} \overline{\mathbf{x y x y}}=0,
$$

which implies that $a_{i}+b_{j}>0$ for all $i \in[m], j \in[n]$. By Theorem 2.1, it yields that the tensor $\mathcal{C}$ is M-positive semi-definite. 
On the other hand, suppose $\mathbf{x}, \overline{\mathbf{x}} \in \mathbb{R}_{+}^{m}, \mathbf{y}, \overline{\mathbf{y}} \in \mathbb{R}_{+}^{n}$, and $\mathbf{x} \geq \overline{\mathbf{x}}$ and $\mathbf{y} \geq \overline{\mathbf{y}}$. From Theorem 2.1, we obtain that $a_{i}+b_{j}>0$ for all $i \in[m], j \in[n]$. Furthermore,

$$
\begin{aligned}
f(\mathbf{x}, \mathbf{y})-f(\overline{\mathbf{x}}, \overline{\mathbf{y}}) & =\mathcal{C} \mathbf{x y \mathbf { x y }}-\mathcal{C} \overline{\mathbf{x y} \mathbf{x y}} \\
& =\sum_{i, k \in[m], j, l \in[n]} c_{i j k l}\left(x_{i} y_{j} x_{k} y_{l}-\bar{x}_{i} \bar{y}_{j} \bar{x}_{k} \bar{y}_{l}\right) \\
& =\sum_{i, k \in[m], j, l \in[n]} \frac{1}{a_{i}+b_{j}+a_{k}+b_{l}}\left(x_{i} y_{j} x_{k} y_{l}-\bar{x}_{i} \bar{y}_{j} \bar{x}_{k} \bar{y}_{l}\right) \\
& \geq 0,
\end{aligned}
$$

which implies that $f(\mathbf{x}, \mathbf{y})$ is monotonically increasing in $\mathbb{R}_{+}^{m} \times \mathbb{R}_{+}^{n}$ and the desired result holds.

Theorem 2.5 Let $\mathcal{C}$ be a fourth-order Cauchy tensor with generating vectors $\mathbf{a} \in \mathbb{R}^{m}$ and $\mathbf{b} \in \mathbb{R}^{n}$. If the tensor $\mathcal{C}$ is $M$-positive definite, then the homogeneous polynomial $f(\mathbf{x}, \mathbf{y})$ is strictly monotone increasing in $\mathbb{R}_{+}^{m} \times \mathbb{R}_{+}^{n}$.

Proof From Theorem 2.3, one has, for all $i \in[m], j \in[n]$,

$$
a_{i}+b_{j}>0
$$

For any $\mathbf{x} \geq \overline{\mathbf{x}}, \mathbf{x} \neq \overline{\mathbf{x}}$ and $\mathbf{y} \geq \overline{\mathbf{y}}, \mathbf{y} \neq \overline{\mathbf{y}}$, then there exist indexes $i_{0} \in[m]$ and $j_{0} \in[n]$ such that

$$
x_{i_{0}}>\bar{x}_{i_{0}} \geq 0
$$

and

$$
y_{j_{0}}>\bar{y}_{j_{0}} \geq 0 \text {. }
$$

Thus,

$$
\begin{aligned}
f(\mathbf{x}, \mathbf{y})-f(\overline{\mathbf{x}}, \overline{\mathbf{y}})= & \mathcal{C} \mathbf{x y \mathbf { x y }}-\mathcal{C} \overline{\mathbf{x y x y}} \\
= & \sum_{i, k \in[m], j, l \in[n]} c_{i j k l}\left(x_{i} y_{j} x_{k} y_{l}-\bar{x}_{i} \bar{y}_{j} \bar{x}_{k} \bar{y}_{l}\right) \\
= & \sum_{i, k \in[m], j, l \in[n],(i, j, k, l) \neq\left(i_{0}, j_{0}, i_{0}, j_{0}\right)} c_{i j k l}\left(x_{i} y_{j} x_{k} y_{l}-\bar{x}_{i} \bar{y}_{j} \bar{x}_{k} \bar{y}_{l}\right) \\
& +c_{i_{0} j_{0} i_{0} j_{0}}\left(x_{i_{0}}^{2} y_{j_{0}}^{2}-\bar{x}_{i_{0}}^{2} \bar{y}_{j_{0}}^{2}\right) \\
= & \sum_{i, k \in[m], j, l \in[n],(i, j, k, l) \neq\left(i_{0}, j_{0}, i_{0}, j_{0}\right)} \frac{1}{a_{i}+b_{j}+a_{k}+b_{l}}\left(x_{i} y_{j} x_{k} y_{l}-\bar{x}_{i} \bar{y}_{j} \bar{x}_{k} \bar{y}_{l}\right) \\
& +\frac{1}{2\left(a_{i_{0}}+b_{j_{0}}\right)}\left(x_{i_{0}}^{2} y_{j_{0}}^{2}-\bar{x}_{i_{0}}^{2} \bar{y}_{j_{0}}^{2}\right) \\
> & 0,
\end{aligned}
$$


which means that the homogeneous polynomial $f(\mathbf{x}, \mathbf{y})$ is strictly monotone increasing in $\mathbb{R}_{+}^{m} \times \mathbb{R}_{+}^{n}$.

Now, we are in a position to propose an example to reveal that the strictly monotone increasing property for the polynomial $f(\mathbf{x}, \mathbf{y})$ is only a necessary condition for the Mpositive definiteness property of the tensor $\mathcal{C}$ but not a sufficient condition.

Example 2.1 Let the tensor $\mathcal{C}=\left(c_{i j k l}\right)$ be a fourth-order Cauchy tensor with generating vectors $\mathbf{a}=(2,2,2)$ and $\mathbf{b}=(4,4,4,4)$. Then one has

$$
c_{i j k l}=\frac{1}{12}, \quad i, k \in[3], j, l \in[4]
$$

and the homogeneous polynomial

$$
f(\mathbf{x}, \mathbf{y})=\mathcal{C} \mathbf{x y x y}=\frac{1}{12} \sum_{i, k \in[3], j, l \in[4]} x_{i} y_{j} x_{k} y_{l}
$$

It is easy to check that $f(\mathbf{x}, \mathbf{y})$ is strictly monotone increasing in $\mathbb{R}_{+}^{3} \times \mathbb{R}_{+}^{4}$. However, it follows from Theorem 2.3 that the tensor $\mathcal{C}$ is not M-positive definite.

Copositive tensors have some important applications in polynomial optimization [18], vacuum stability of a general scalar potential [30], tensor generalized eigenvalue complementarity problem [31], and tensor complementarity problem [32,33]. The tensor $\mathcal{C}$ is called copositive if $\mathcal{C} \mathbf{x}^{m} \geq 0$ for all $\mathbf{x} \in \mathbb{R}_{+}^{n}$. Consider the tensor complement problem $(\mathrm{TCP}(\mathbf{q}, \mathcal{C}))$ of finding a vector $\mathbf{x} \in \mathbb{R}^{n}$ such that

$$
\mathbf{x} \geq \mathbf{0}, \quad \mathbf{q}+\mathcal{C} \mathbf{x}^{m-1} \geq \mathbf{0}, \quad \mathbf{x}^{T}\left(\mathbf{q}+\mathcal{C} \mathbf{x}^{m-1}\right)=0
$$

Applying the above definitions, we have the following technical conclusion.

Theorem 2.6 Let $\mathcal{C}$ be a fourth-order Cauchy tensor with generating vectors $\mathbf{a}, \mathbf{b} \in \mathbb{R}_{+}^{n}$. Then the following statements are equivalent:

(i) the tensor $\mathcal{C}$ is copositive;

(ii) for every $\mathbf{q}>\mathbf{0}, \mathrm{TCP}(\mathbf{q}, \mathcal{C})$ has a unique solution;

(iii) for every index set $N \subset[n]$, the system

$$
\mathcal{C}^{|N|}\left(\mathbf{x}^{N}\right)^{3}<\mathbf{0}, \quad \mathbf{x}^{N} \geq \mathbf{0}
$$

has no solution, where $\mathbf{x}^{N} \in \mathbb{R}^{|N|}$;

(iv) for all $i \in[n], a_{i}+b_{i}>0$.

Proof (i) $\Rightarrow$ (ii). It follows from $\mathbf{q}>\mathbf{0}$ that $\mathbf{0}$ is a solution of $\operatorname{TCP}(\mathbf{q}, \mathcal{C})$. Suppose, to the contrary, that there exists a vector $\mathbf{q}^{\prime}>\mathbf{0}$ such that $\operatorname{TCP}\left(\mathbf{q}^{\prime}, \mathcal{C}\right)$ has a solution $\mathbf{x} \neq \mathbf{0}$. Since $\mathcal{C}$ is copositive, for $\mathbf{x} \in \mathbb{R}_{+}^{n}$, one has

$$
\mathcal{C} \mathbf{x}^{4} \geq 0
$$


On the other hand,

$$
\begin{aligned}
\left(\mathcal{C} \mathbf{x}^{3}\right)_{i} & =\sum_{k, j, k \in[n]} c_{i j k l} x_{j} x_{k} x_{l} \\
& =\sum_{k, j, k \in[n]} \frac{x_{j} x_{k} x_{l}}{a_{i}+b_{j}+a_{k}+b_{l}} \\
& \geq \mathbf{0},
\end{aligned}
$$

which means $\mathbf{q}^{\prime}+\mathcal{C} \mathbf{x}^{3}>\mathbf{0}$. Then

$$
\mathbf{x}^{T}\left(\mathbf{q}^{\prime}+\mathcal{C} \mathbf{x}^{3}\right)=\mathbf{x}^{T} \mathbf{q}^{\prime}+\mathcal{C} \mathbf{x}^{4}>0
$$

which contradicts the assumption that $x$ solves $\operatorname{TCP}\left(\mathbf{q}^{\prime}, \mathcal{C}\right)$. Thus, for every $\mathbf{q}>\mathbf{0}$, we obtain $\operatorname{TCP}(\mathbf{q}, \mathcal{C})$ has a unique solution.

(ii) $\Rightarrow$ (iii). Following the proof of Theorem 3.1 [34], we have the desired result.

(iii) $\Rightarrow$ (iv). Let $N=\{i\}, i \in[n], \mathbf{x}^{N}=1$. Then

$$
\left(\mathcal{C}^{|N|}\left(\mathbf{x}^{N}\right)^{3}\right)_{i}=c_{i i i i} x_{i}^{3}=c_{i i i i}=\frac{1}{2\left(a_{i}+b_{i}\right)}>0 .
$$

Then, for all $i \in[n]$, one has $a_{i}+b_{i}>0$.

(iv) $\Rightarrow$ (i). By Theorem 2.1, we obtain that the tensor $\mathcal{C}$ is M-positive semi-definite, which means that the tensor $\mathcal{C}$ is copositive.

\section{Spectral properties for fourth-order Cauchy tensors}

In this section, we discuss M-eigenvalue inclusion theorems and spectral properties of fourth-order Cauchy tensors. M-eigenvalue problem has a close relationship with the strong ellipticity condition, which plays an important role in nonlinear elasticity and in materials, since it can ensure an elastic material to satisfy some mechanical properties. Thus, to identify whether the strong ellipticity condition of a given material holds or not becomes an important problem in mechanics [29, 35-38].

The strong ellipticity condition for a partially symmetric fourth-order tensor $\mathcal{C}$ is stated by

$$
f(\mathbf{x}, \mathbf{y})=\mathcal{C} \mathbf{x y x y}=\sum_{i, k, j, l \in[n]} c_{i j k l} x_{i} y_{j} x_{k} y_{l}>0
$$

for any vectors $\mathbf{x} \in \mathbb{R}^{n}, \mathbf{x} \neq 0, \mathbf{y} \in \mathbb{R}^{n}, \mathbf{y} \neq 0$. In [39], Qi, Dai, and Han revealed the necessary and sufficient condition of the strong ellipticity condition by introducing the following definition of an M-eigenvalue of the tensor $\mathcal{C}$. For $\lambda \in \mathbb{R}, \mathbf{x} \in \mathbb{R}^{m}, \mathbf{y} \in \mathbb{R}^{n}$, it holds that

$$
\left\{\begin{array}{l}
\mathcal{C} \cdot \mathbf{y x y}=\lambda \mathbf{x}, \\
\mathcal{C} \mathbf{x y x} \cdot=\lambda \mathbf{y}, \\
\mathbf{x}^{T} \mathbf{x}=1, \\
\mathbf{y}^{T} \mathbf{y}=1,
\end{array}\right.
$$


where $(\mathcal{C} \cdot \mathbf{y x y})_{i}=\sum_{k \in[m], j, l \in[n]} c_{i j k l} y_{j} x_{k} y_{l}$, and $(\mathcal{C} \mathbf{x y x} \cdot)_{l}=\sum_{i, k \in[m], j \in[n]} c_{i j k l} x_{i} y_{j} x_{k}$. The scalar $\lambda$ is called an M-eigenvalue of the tensor $\mathcal{C}$, and $\mathbf{x}$ and $\mathbf{y}$ are called left and right Meigenvectors of $\mathcal{C}$, respectively, associated with the M-eigenvalue $\lambda$. When $m=n$, Qi, Dai, and Han [39] gave the following technical theorem.

Theorem 3.1 ([39]) The strong ellipticity condition holds if and only if the smallest Meigenvalue of the elasticity tensor is positive.

From Theorems 2.3 and 3.1, we can obtain the necessary and sufficient conditions of the strong ellipticity condition for fourth-order Cauchy tensors.

Theorem 3.2 Let the vectors $\mathbf{a} \in \mathbb{R}^{n}, \mathbf{b} \in \mathbb{R}^{n}$ be generating vectors of the fourth-order Cauchy tensor $\mathcal{C}$. The strong ellipticity condition holds if and only if the smallest $M$ eigenvalue of the tensor $\mathcal{C}$ is positive.

Theorem 3.3 Let the vectors $\mathbf{a} \in \mathbb{R}^{n}, \mathbf{b} \in \mathbb{R}^{n}$ be generating vectors of the fourth-order Cauchy tensor $\mathcal{C}$. The strong ellipticity condition holds if and only if $a_{i}+b_{j}>0$ for all $i, j \in[n]$, and the elements of generating vectors $a, b$ are mutually distinct, respectively.

The spectral radius $\rho(\mathcal{C})$ of $\mathcal{C}$ is defined as

$$
\rho(\mathcal{C})=\max \{|\lambda|: \lambda \in \sigma(\mathcal{C})\}
$$

where $\sigma(\mathcal{C})$ is the spectrum of $\mathcal{C}$, which contains all M-eigenvalues of $\mathcal{C}$.

Now, inspired by the idea of $\mathrm{H}$-eigenvalue inclusion theorem [40], we establish the following M-eigenvalue inclusion theorems for fourth-order Cauchy tensors.

Theorem 3.4 Suppose that the tensor $\mathcal{C}$ is a fourth-order Cauchy tensor with generating vectors $\mathbf{a} \in \mathbb{R}^{m}, \mathbf{b} \in \mathbb{R}^{n}$. Then

$$
\sigma(\mathcal{C}) \subseteq \Gamma(\mathcal{C})=\bigcup_{i \in[m]} \Gamma_{i}(\mathcal{C})
$$

where $\Gamma_{i}(\mathcal{C})=\left\{z \in \mathbb{C}:|z| \leq \sum_{k \in[m], j, l \in[n]} \frac{1}{a_{i}+b_{j}+a_{k}+b_{l}}\right\}$.

Proof Let $\lambda$ be an M-eigenvalue of the tensor $\mathcal{C}$ with left $M$-eigenvector $\mathbf{x} \in \mathbb{R}^{m}$ and right M-eigenvector $\mathbf{y} \in \mathbb{R}^{n}$. Since $\mathbf{x}$ is a left M-eigenvector of the tensor $\mathcal{C}$ with $\mathbf{x}^{T} \mathbf{x}=1$, we know that it has at least one nonzero component. Assume that

$$
\left|x_{t}\right|=\max _{i \in[m]}\left|x_{i}\right|>0
$$

It follows from (3.1) that

$$
\begin{aligned}
\lambda x_{t} & =(\mathcal{C} \cdot \mathbf{y x y})_{t} \\
& =\sum_{k \in[m], j, l \in[n]} c_{t j k l} y_{j} x_{k} y_{l} \\
& =\sum_{k \in[m], j, l \in[n]} \frac{y_{j} x_{k} y_{l}}{a_{t}+b_{j}+a_{k}+b_{l}} .
\end{aligned}
$$


Since $\mathbf{y}^{T} \mathbf{y}=1$, we obtain $\left|y_{j}\right| \leq 1$ for any $j \in[n]$. Furthermore,

$$
\begin{aligned}
|\lambda| & \leq \sum_{k \in[m], j, l \in[n]} \frac{1}{\left|a_{t}+b_{j}+a_{k}+b_{l}\right|} \frac{\left|x_{k}\right|}{\left|x_{t}\right|}\left|y_{j} y_{l}\right| \\
& \leq \sum_{k \in[m], j, l \in[n]} \frac{1}{\left|a_{t}+b_{j}+a_{k}+b_{l}\right|} .
\end{aligned}
$$

This implies that $\lambda \in \Gamma(\mathcal{C})$.

Theorem 3.5 Suppose that the tensor $\mathcal{C}$ is a fourth-order Cauchy tensor with generating vectors $\mathbf{a} \in \mathbb{R}^{m}, \mathbf{b} \in \mathbb{R}^{n}$. If there exists an index $i \in[\mathrm{m}]$ such that $c_{i 1 i 1}=c_{i 2 i 2}=\cdots=c_{\text {inin }}=d$, then

$$
\sigma(\mathcal{C}) \subseteq \mathcal{K}(\mathcal{C})=\bigcup_{i \in[m]} \mathcal{K}_{i}(\mathcal{C})
$$

where $\mathcal{K}_{i}(\mathcal{C})=\left\{z \in \mathbb{C}:|z-d| \leq \sum_{j, l \in[n], j \neq l} \frac{1}{\left|a_{i}+b_{j}+a_{i}+b_{l}\right|}+\sum_{k \neq i, k \in[m], j, l \in[n]} \frac{1}{a_{i}+b_{j}+a_{k}+b_{l}}\right\}$

Proof Let $\lambda$ be an M-eigenvalue of the tensor $\mathcal{C}$ with corresponding left M-eigenvector $\mathbf{x} \in$ $\mathbb{R}^{m}$ with $\mathbf{x}^{T} \mathbf{x}=1$ and right M-eigenvector $\mathbf{y} \in \mathbb{R}^{n}$ with $\mathbf{y}^{T} \mathbf{y}=1$. Then left M-eigenvector $\mathbf{x}$ has at least one nonzero component. Assume that

$$
\left|x_{t}\right|=\max _{i \in[m]}\left|x_{i}\right|>0 .
$$

It follows from (3.1) that

$$
\begin{aligned}
\lambda x_{t} & =(\mathcal{C} \cdot \mathbf{y x y})_{t} \\
& =\sum_{k \in[m], j, l \in[n]} c_{t j k l} y_{j} x_{k} y_{l} \\
& =\sum_{j \in[n]} c_{t i j j} y_{j}^{2} x_{t}+\sum_{j, l \in[n], j \neq l} c_{t j t l} y_{j} x_{t} y_{l}+\sum_{k \neq t, k \in[m], j, l \in[n]} c_{t j k l} y_{j} x_{k} y_{l} \\
& =d x_{t}+\sum_{j, l \in[n], j \neq l} c_{t j t l} y_{j} x_{t} y_{l}+\sum_{k \neq t, k \in[m], j, l \in[n]} c_{t j k l} y_{j} x_{k} y_{l} .
\end{aligned}
$$

Furthermore,

$$
\begin{aligned}
|\lambda-d| & \leq \sum_{j, l \in[n], j \neq l} \frac{1}{\left|a_{t}+b_{j}+a_{t}+b_{l}\right|}\left|y_{j} y_{l}\right|+\sum_{k \neq t, k \in[m], j, l \in[n]} \frac{1}{\left|a_{t}+b_{j}+a_{k}+b_{l}\right|} \frac{\left|x_{k}\right|}{\left|x_{t}\right|}\left|y_{j} y_{l}\right| \\
& \leq \sum_{j, l \in[n], j \neq l} \frac{1}{\left|a_{t}+b_{j}+a_{t}+b_{l}\right|}+\sum_{k \neq t, k \in[m], j, l \in[n]} \frac{1}{\left|a_{t}+b_{j}+a_{k}+b_{l}\right|} .
\end{aligned}
$$

This implies that $\lambda \in \mathcal{K}(\mathcal{C})$.

Next, we will reveal several spectral properties for fourth-order Cauchy tensors. 
Theorem 3.6 Suppose that the tensor $\mathcal{C}$ is a fourth-order Cauchy tensor with generating vectors $\mathbf{a} \in \mathbb{R}^{m}, \mathbf{b} \in \mathbb{R}^{n}$, and for all $i \in[m], j \in[n]$ such that $a_{i}+b_{j}>0$. Then the tensor $\mathcal{C}$ is M-positive definite if and only if its $M$-eigenvalues are positive.

Proof Suppose that $\lambda, \mathbf{x}$, and $\mathbf{y}$ satisfy (3.1). It is easy to obtain that $\lambda=\mathcal{C} \mathbf{x y x y}$. Furthermore, (3.1) is the optimality condition of

$$
\min \left\{\mathcal{C} \mathbf{x y x y}: \mathbf{x}^{T} \mathbf{x}=1, \mathbf{y}^{T} \mathbf{y}=1, \mathbf{x} \in \mathbb{R}^{m}, \mathbf{y} \in \mathbb{R}^{n}\right\}
$$

From the feasible set is compact and the objective function is continuous, we obtain that the global maximizer and minimizer always exist. This shows that $\mathcal{C}$ always has $\mathrm{M}$ eigenvalues. Since $\mathcal{C}$ is M-positive definite (M-positive semidefinite) if and only if the optimal value of (3.2) is positive (nonnegative), we obtain the desired result.

Theorem 3.7 Suppose that the fourth-order Cauchy tensor $\mathcal{C}$ and its generating vectors a, $\mathbf{b}$ are defined as in Theorem 3.6 with the elements of generating vectors $\mathbf{a}, \mathbf{b}$ mutually distinct, respectively. If $\lambda \in \sigma(\mathcal{C})$ is an $M$-eigenvalue of the tensor $\mathcal{C}$ with non-negative left $M$-eigenvector $\mathbf{x}$ or non-negative right $M$-eigenvector $\mathbf{y}$, then $\lambda \neq 0$.

Proof Since $\mathbf{x} \geq \mathbf{0}$ and $\mathbf{x} \neq \mathbf{0}$, we know that it has at least one nonzero component $x_{t}>0$. From the definitions of $\mathrm{M}$-eigenvalue and $\mathrm{M}$-eigenvector of the tensor $\mathcal{C}$, one has

$$
\begin{aligned}
\lambda x_{i} & =(\mathcal{C} \cdot \mathbf{y x y})_{i} \\
& =\sum_{k \in[m], j, l \in[n]} \frac{y_{j} x_{k} y_{l}}{a_{t}+b_{j}+a_{k}+b_{l}} \\
& =\sum_{k \in[m], j, l \in[n]} \int_{0}^{1} t^{a_{t}+b_{j}+a_{k}+b_{l}-1} y_{j} x_{k} y_{l} d t \\
& =\int_{0}^{1} t^{a_{t}-\frac{1}{4}}\left(\sum_{k \in[m]} t^{a_{k}-\frac{1}{4}} x_{k}\right)\left(\sum_{j \in[n]} t^{b_{j}-\frac{1}{4}} y_{j}\right)^{2} d t .
\end{aligned}
$$

Suppose, to the contrary, that the tensor $\mathcal{C}$ has M-eigenvalue $\lambda=0$ with left Meigenvector $\mathbf{x}$. Then, from (3.3), we have

$$
\int_{0}^{1} t^{a_{t}-\frac{1}{4}}\left(\sum_{k \in[m]} t^{a_{k}-\frac{1}{4}} x_{k}\right)\left(\sum_{j \in[n]} t^{b_{j}-\frac{1}{4}} y_{j}\right)^{2} d t \equiv 0 .
$$

Using the properties of integration, one has

$$
\left(\sum_{k \in[m]} t^{a_{k}-\frac{1}{4}} x_{k}\right)\left(\sum_{j \in[n]} t^{b_{j}-\frac{1}{4}} y_{j}\right)^{2} \equiv 0, \quad t \in(0,1],
$$

which implies

$$
\sum_{k \in[m]} t^{a_{k}-\frac{1}{4}} x_{k} \equiv 0, \quad t \in(0,1],
$$


or

$$
\sum_{j \in[n]} t^{b_{j}-\frac{1}{4}} y_{j} \equiv 0, \quad t \in(0,1] .
$$

Without loss of generality, we assume

$$
\sum_{k \in[m]} t^{a_{k}-\frac{1}{4}} x_{k} \equiv 0, \quad t \in(0,1] .
$$

Furthermore,

$$
x_{1}+t^{a_{2}-a_{1}} x_{2}+\cdots+t^{a_{m}-a_{1}} x_{m} \equiv 0, \quad t \in(0,1] .
$$

By the continuity and the fact that $a_{1}, a_{2}, \ldots, a_{m}$ are mutually distinct, it yields that

$$
x_{1}=0
$$

and

$$
x_{2}+t^{a_{3}-a_{2}} x_{3}+\cdots+t^{a_{m}-a_{2}} x_{m} \equiv 0, \quad t \in(0,1] .
$$

Applying the same argument, one has

$$
x_{1}=x_{2}=\cdots=x_{m}=0 \text {, }
$$

which contradicts with $\mathbf{x}^{T} \mathbf{x}=1$. Then the tensor $\mathcal{C}$ has no zero M-eigenvalue. Similarly, if right $M$-eigenvector $\mathbf{y} \geq \mathbf{0}$, using the second equation of (3.1), we can also obtain that the tensor $\mathcal{C}$ has no zero $\mathrm{M}$-eigenvalue and the desired conclusion follows.

\section{Power method of fourth-order Cauchy tensors}

In this section, a power method is proposed to compute the smallest and the largest Meigenvalues of fourth-order Cauchy tensors. It is well known that the power method is an efficient method to solve the largest eigenvalue of a matrix [41]. The method has successfully extended to compute the largest Z-eigenvalue in magnitude of higher-order tensors [40] and the largest M-eigenvalue of a fourth-order partially symmetric tensor [42]. Motivated by these, we first propose a power method to compute the smallest M-eigenvalue of fourth-order Cauchy tensors.

We introduce the following identity tensor $\mathcal{I} \in \mathbb{R}^{m \times n \times m \times n}$ :

$$
\mathcal{I}_{i j k l}= \begin{cases}1, & \text { if } i=k \text { and } j=l, \\ 0, & \text { otherwise. }\end{cases}
$$

Choose a suitable $\alpha \in \mathbb{R}$ such that $\alpha>|\lambda|$, where $\lambda \in \sigma(\mathcal{C})$, and take

$$
\bar{f}(\mathbf{x}, \mathbf{y})=\alpha \mathcal{I} \mathbf{x y x y}-\mathcal{C} \mathbf{x y x y} \triangleq \overline{\mathcal{C}} \mathbf{x y x y} .
$$


It is easy to check that the tensor $\overline{\mathcal{C}}$ is M-positive definite on $\mathbb{R}^{m} \times \mathbb{R}^{n}$ with the same symmetry property of the tensor $\mathcal{C}$. Moreover, Theorem 3.4 suggests that we can take

$$
\alpha=(1+\varepsilon) \max _{i \in[m]} \sum_{k \in[m], j, l \in[n]}\left|c_{i j k l}\right|
$$

where $\varepsilon>0$ is a sufficiently small number. Furthermore, if $\mathbf{x}$ and $\mathbf{y}$ constitute a pair of Meigenvectors of the tensor $\overline{\mathcal{C}}$ associated with M-eigenvalue $\lambda$, then they are also a pair of M-eigenvectors of tensor $\mathcal{C}$ associated with M-eigenvalue $\alpha-\lambda$.

Now, we are in a position to propose a power method to compute the smallest Meigenvalue of a fourth-order Cauchy tensor $\mathcal{C}$.

\section{Algorithm 4.1}

Initialization step: Choose initial points $\mathbf{x}_{0} \in \mathbb{R}^{m}, \mathbf{y}_{0} \in \mathbb{R}^{n}$, and let $k=0$.

Iterative step: Carry out the following formula alternatively until certain convergence criterion is satisfied and output $\mathbf{x}^{*}, \mathbf{y}^{*}$ :

$$
\begin{aligned}
& \bar{x}_{k+1}=\overline{\mathcal{C}} \cdot y_{k} x_{k} y_{k}, \quad x_{k+1}=\frac{\bar{x}_{k+1}}{\left\|\bar{x}_{k+1}\right\|}, \\
& \bar{y}_{k+1}=\overline{\mathcal{C}} x_{k+1} y_{k} x_{k+1}, \quad y_{k+1}=\frac{\bar{y}_{k+1}}{\left\|\bar{y}_{k+1}\right\|}, \\
& k=k+1 .
\end{aligned}
$$

Final step: Output the smallest M-eigenvalue $\alpha-\bar{f}\left(\mathbf{x}^{*}, \mathbf{y}^{*}\right)$ of the tensor $\mathcal{C}$ and the associated M-eigenvectors $\mathbf{x}^{*}, \mathbf{y}^{*}$.

Similarly, we can compute the largest M-eigenvalue of fourth-order Cauchy tensors. Take

$$
\alpha=(1+\varepsilon) \max _{i \in[m]} \sum_{k \in[m], j, l \in[n]}\left|c_{i j k l}\right|
$$

and define

$$
\widehat{f}(x, y)=\alpha \mathcal{I} \mathbf{x y x y}+\mathcal{C} \mathbf{x y x y} \triangleq \widehat{\mathcal{C}} \mathbf{x y x y} .
$$

Obviously, if $\mathbf{x}$ and $\mathbf{y}$ constitute a pair of M-eigenvectors of the tensor $\widehat{\mathcal{C}}$ associated with M-eigenvalue $\widehat{\lambda}$, then they are also a pair of M-eigenvectors of the tensor $\mathcal{C}$ associated with M-eigenvalue $\widehat{\lambda}-\alpha$. Thus, we can apply Algorithm 4.1 to compute the largest Meigenvalue of a fourth-order Cauchy tensor $\mathcal{C}$.

The following numerical experiments show the effectiveness of the proposed method. The whole program was written in Matlab 7.0. All the numerical results were carried out on a personal Lenovo Thinkpad computer with Intel(R) Core(TM) i7-6500U CPU 2.50 GHz and RAM 8.00 GB. In the implementation, we choose $\left\|x_{k+1}-x_{k}\right\|+\left\|y_{k+1}-y_{k}\right\| \leq$ $10^{-10}$ as the stopping criterion, and take the parameter $\varepsilon=0.0001$. 

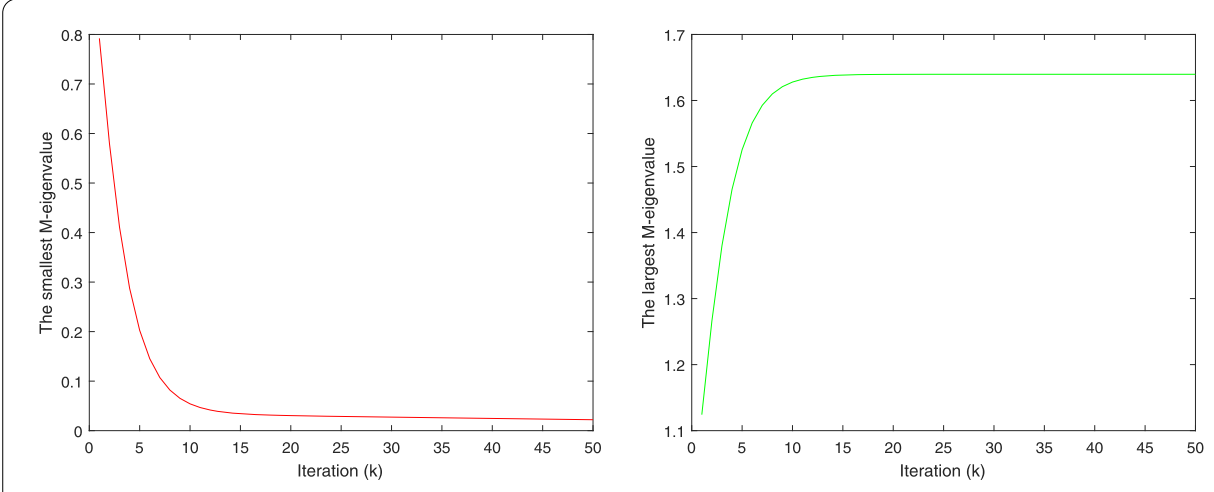

Figure 1 Numerical result of Example 4.1
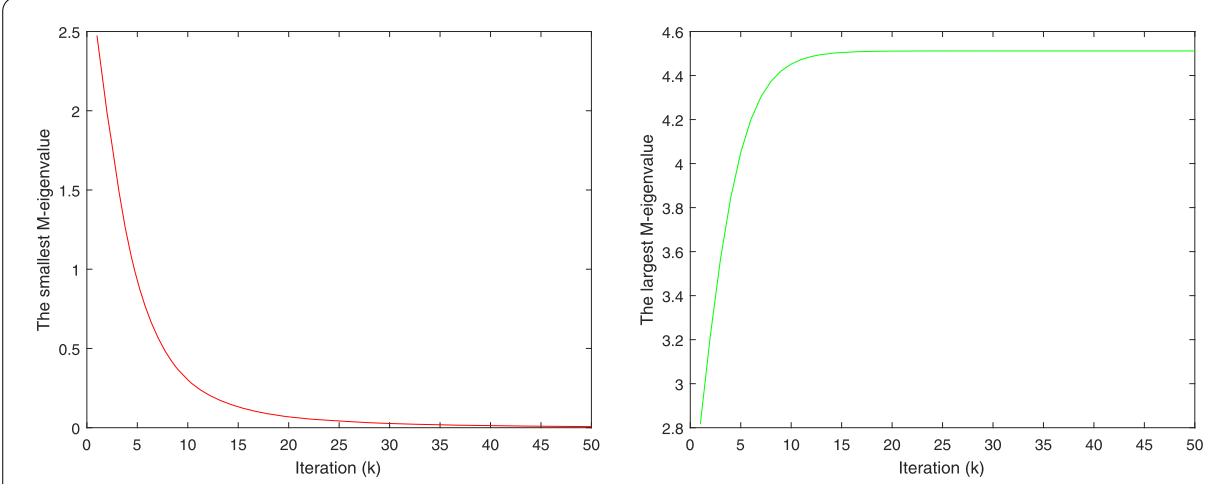

Figure 2 Numerical result of Example 4.2

Example 4.1 Consider a fourth-order Cauchy tensor $\mathcal{C}$ with generating vectors $\mathbf{a}=[4,5,3]$ and $\mathbf{b}=[-1,-2,1]$. The variation of the objective function value corresponding to the tensor $\mathcal{C}$ during the iteration process can be shown in Fig. 1. For the tensor $\mathcal{C}$, its smallest M-eigenvalue is 0.0221 and the largest M-eigenvalue is 1.6397.

Example 4.2 Consider a fourth-order Cauchy tensor $\mathcal{C}$ with generating vectors $\mathbf{a}=$ $[1.4424,1.1837,1.2492]$ and $\mathbf{b}=[0.0285,0.0530,0.0560,0.0415]$. The variation of the objective function value corresponding to the tensor $\mathcal{C}$ during the iteration process can be shown in Fig. 2. For the tensor $\mathcal{C}$, its smallest M-eigenvalue is 0.0069 and the largest Meigenvalue is 4.5117 .

Example 4.3 Consider a fourth-order Cauchy tensor $\mathcal{C}$ with generating vectors $\mathbf{a}=$ $\operatorname{rand}(20,1)+10$ and $\mathbf{b}=30 * \operatorname{rand}(30,1)$. The variation of the objective function value corresponding to the tensor $\mathcal{C}$ during the iteration process can be shown in Fig. 4. For the tensor $\mathcal{C}$, its smallest $\mathrm{M}$-eigenvalue is 0.0594 and the largest $\mathrm{M}$-eigenvalue is 12.4184 .

Example 4.4 Consider a fourth-order Cauchy tensor $\mathcal{C}$ with generating vectors $\mathbf{a}=5 *$ $\operatorname{rand}(30,1)+10$ and $\mathbf{b}=\operatorname{rand}(40,1)+8$. The variation of the objective function value corresponding to the tensor $\mathcal{C}$ during the iteration process can be shown in Fig. 4. For the tensor $\mathcal{C}$, its smallest $\mathrm{M}$-eigenvalue is 0.0229 and the largest M-eigenvalue is 28.9232 . 

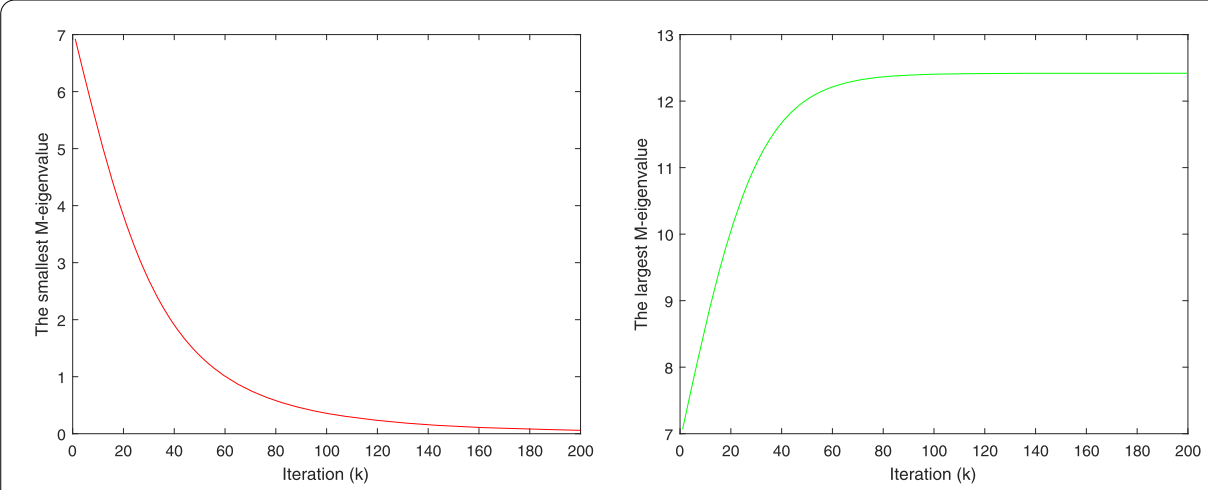

Figure 3 Numerical result of Example 4.3
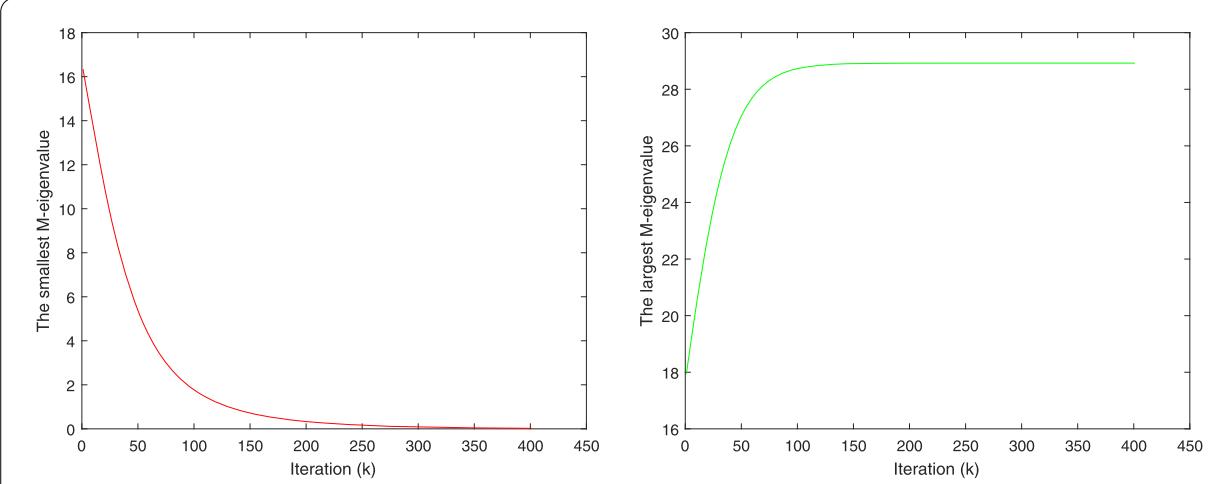

Figure 4 Numerical result of Example 4.4

Figures 1, 2, 3, and 4 show that the smallest M-eigenvalue sequence generated by Algorithm 4.1 is decreasing, and the largest M-eigenvalue sequence generated by Algorithm 4.1 is nondecreasing. From Theorems 3.2 and 3.3, it is easy to see that M-eigenvalues always exist and the strong ellipticity condition holds if and only if the smallest M-eigenvalue of $\mathcal{C}$ is positive; thus Example 4.1 verifies the strong ellipticity condition. From Theorem 2.3, we know that the tensor $\mathcal{C}$ is M-positive definite if it satisfies that $a_{i}+b_{j}>0$ for all $i, j \in[n]$ and the elements of generating vectors $\mathbf{a}, \mathbf{b}$ are mutually distinct, respectively; then Example 4.2 shows that the tensor $\mathcal{C}$ is $\mathrm{M}$-positive definite. Furthermore, Examples 4.3 and 4.4 reclaim that our algorithm is also suitable for the tensors with high dimensions.

\section{Final remarks}

In this article, the necessary and sufficient conditions for the M-positive semi-definiteness and M-positive definiteness of fourth-order Cauchy tensors are discussed. Moreover, the necessary and sufficient conditions of the strong ellipticity condition for fourth-order Cauchy tensors are obtained. Furthermore, we reveal that fourth-order Cauchy tensors are M-positive semi-definite if and only if there is a monotone increasing homogeneous polynomial defined in the nonnegative orthant of $\mathbb{R}^{m} \times \mathbb{R}^{n}$. Several M-eigenvalue inclusion theorems and spectral properties of fourth-order Cauchy tensors are discussed. A power method is proposed to compute the smallest and the largest M-eigenvalues of fourth-order 
Cauchy tensors. The given numerical experiments show the effectiveness of the proposed method.

However, there are still some questions that we are not sure about now. Can we have the type of Cauchy-Toeplitz tensors with the partially symmetric property? If so, how about their spectral properties? What are the necessary and sufficient conditions for their M-positive semi-definiteness?

\section{Acknowledgements}

Haibin Chen's research was done during his postdoctoral period in Qufu Normal University. We are thankful to Dr. Min Sun for his valuable comments to the numerical experiments.

\section{Funding}

This work was supported by the Natural Science Foundation of China $(11401438,11671228,11601261,11571120)$, Shandong Provincial Natural Science Foundation (ZR2016AQ12), and Project of Shandong Province Higher Educational Science and Technology Program (Grant No. J14LI52).

\section{Competing interests}

The authors declare that they have no competing interests.

\section{Authors' contributions}

All authors contributed equally to the manuscript. All authors read and approved the final manuscript.

\section{Author details}

${ }^{1}$ School of Mathematics and Information Science, Weifang University, Weifang, China. ${ }^{2}$ School of Management Science, Qufu Normal University, Rizhao, China.

\section{Publisher's Note}

Springer Nature remains neutral with regard to jurisdictional claims in published maps and institutional affiliations.

Received: 23 July 2018 Accepted: 27 January 2019 Published online: 04 February 2019

\section{References}

1. Walton, J.R., Wilber, J.P.: Sufficient conditions for strong ellipticity for a class of anisotropic materials. Int. J. Non-Linear Mech. 38, 411-455 (2003)

2. Pólya, G., Szegö, G.: Zweiter Band. Springer, Berlin (1925)

3. Finck, T., Heinig, G., Rost, K.: An inversion formula and fast algorithms for Cauchy-Vandermonde matrices. Linear Algebra Appl. 183, 179-191 (1993)

4. Gohberg, l., Olshevsky, V.: Fast algorithms with preprocessing for matrix-vector multiplication problems. J. Complex. 10, 411-427 (1994)

5. Heinig, G.: Inversion of generalized Cauchy matrices and other classes of structured matrices. In: Linear Algebra for Signal Processing, pp. 63-81. Springer, New York (1995)

6. Chen, H., Qi, L.: Positive definiteness and semi-definiteness of even order symmetric Cauchy tensors. J. Ind. Manag. Optim. 11, 1263-1274 (2017)

7. Chen, H., Li, G., Qi, L.: Further results on Cauchy tensors and Hankel tensors. Appl. Math. Comput. 275, 50-62 (2016)

8. Chen, H., Li, G., Qi, L.: SOS tensor decomposition: theory and applications. Commun. Math. Sci. 14, 2073-2100 (2015)

9. Ding, W., Qi, L., Wei, Y.: M-tensors and nonsingular M-tensors. Linear Algebra Appl. 439, 3264-3278 (2013)

10. Ding, W., Qi, L., Wei, Y.: Fast Hankel tensor-vector products and application to exponential data fitting. Numer. Linear Algebra Appl. 10, 70-78 (2014)

11. Qi, L.: Hankel tensors: associated Hankel matrices and Vandermonde decomposition. Commun. Math. Sci. 13, 113-125 (2013)

12. Qi, L., Song, Y.: An even order symmetric B tensor is positive definite. Linear Algebra Appl. 457, 303-312 (2014)

13. Qi, L., Xu, C., Xu, Y.: Nonnegative tensor factorization, completely positive tensors and a hierarchical elimination algorithm. SIAM J. Matrix Anal. Appl. 35, 1227-1241 (2014)

14. Song, Y., Qi, L.: Some properties of infinite and finite dimension Hilbert tensors. Linear Algebra Appl. 451, 1-14 (2014)

15. Song, Y., Qi, L.: Properties of some classes of structured tensors. J. Optim. Theory Appl. 3, 1-20 (2015)

16. Yuan, P., You, L.: Some remarks on $P_{,} P_{0}, B$ and $B_{0}$ tensors. Linear Algebra Appl. 3, 511-521 (2014)

17. Zhang, L., Qi, L., Zhou, G.: M-tensors and some applications. SIAM J. Matrix Anal. Appl. 35, 437-452 (2014)

18. Chen, H., Huang, Z., Qi, L.: Copositivity detection of tensors: theory and algorithm. J. Optim. Theory Appl. 174 746-761 (2017)

19. Chen, H., Chen, Y., Li, G., Qi, L.: A semi-definite program approach for computing the maximum eigenvalue of a class of structured tensors and its applications in hypergraphs and copositivity test. Numer. Linear Algebra Appl. 25, e2125 (2018)

20. Chen, H., Huang, Z., Qi, L.: Copositive tensor detection and its applications in physics and hypergraphs. Comput. Optim. Appl. 69, 133-158 (2018)

21. Chen, H., Wang, Y.: On computing minimal H-eigenvalue of sign-structured tensors. Front. Math. China 12, 1289-1302 (2017) 
22. Chen, H., Qi, L., Song, Y.: Column sufficient tensors and tensor complementarity problems. Front. Math. China 13(2), 255-276 (2018)

23. Wang, Y., Caccetta, L., Zhou, G.: Convergence analysis of a block improvement method for polynomial optimization over unit spheres. Numer. Linear Algebra Appl. 22, 1059-1076 (2015)

24. Wang, Y., Zhang, K., Sun, H.: Criteria for strong H-tensors. Front. Math. China 11, 577-592 (2016)

25. Zhou, G., Wang, G., Qi, L., Alqahtani, M.: A fast algorithm for the spectral radii of weakly reducible nonnegative tensors. Numer. Linear Algebra Appl. 25(2), e2134 (2018)

26. Zhang, K., Wang, Y:: An H-tensor based iterative scheme for identifying the positive definiteness of multivariate homogeneous forms. J. Comput. Appl. Math. 305, 1-10 (2016)

27. Wang, X., Chen, H., Wang, Y.: Solution structures of tensor complementarity problem. Front. Math. China (2018). https://doi.org/10.1007/s11464-018-0675-2

28. Chen, H., Wang, Y.: High-order copositive tensors and its applications. J. Appl. Anal. Comput. 8(6), 1863-1885 (2018)

29. Chen, Z., Qi, L.: Circulant tensors with applications to spectral hypergraph theory and stochastic process. J. Ind. Manag. Optim. 12, 1227-1247 (2014)

30. Kannike, K.: Vacuum stability of a general scalar potential of a few fields. Eur. Phys. J. C 76, 324 (2016)

31. Ling, C., He, H., Qi, L.: On the cone eigenvalue complementarity problem for higher-order tensors. Comput. Optim. Appl. 63(1), 143-168 (2016)

32. Bai, X., Huang, Z., Wang, Y.: Global uniqueness and solvability for tensor complementarity problems. J. Optim. Theory Appl. 170, $72-84$ (2016)

33. Che, M., Qi, L., Wei, Y.: Positive definite tensors to nonlinear complementarity problems. J. Optim. Theory Appl. 168, 475-487 (2016)

34. Song, Y., Qi, L.: Tensor complementarity problem and semi-positive tensors. J. Optim. Theory Appl. 3, 1069-1078 (2016)

35. Gurtin, M.E.: The linear theory of elasticity. In: Linear Theories of Elasticity and Thermoelasticity, pp. 1-295. Springer, Berlin (1973)

36. Huang, Z., Qi, L.: Positive definiteness of paired symmetric tensors and elasticity tensors. J. Comput. Appl. Math. 338, 22-43 (2018)

37. Ding, W., Qi, L., Yan, H.: On some sufficient conditions for strong ellipticity (2017). arXiv:1705.05081. arXiv preprint

38. Ding, W., Liu, J., Qi, L.: Elasticity M-tensors and the strong ellipticity condition (2017) arXiv preprint. arXiv:1705.09911

39. Qi, L., Dai, H., Han, D.: Conditions for strong ellipticity and M-eigenvalues. Front. Math. China 4, 349-364 (2009)

40. Qi, L.: Eigenvalues of a real supersymmetric tensor. J. Symb. Comput. 6, 1302-1324 (2005)

41. Golub, G.H., Van, C.F.: Matrix Computations, 3rd edn. Johns Hopkins University Press, Baltimore (1996)

42. Wang, Y., Qi, L., Zhang, X.: A practical method for computing the largest M-eigenvalue of a fourth-order partially symmetric tensor. Numer. Linear Algebra Appl. 16, 589-601 (2009)

\section{Submit your manuscript to a SpringerOpen ${ }^{\circ}$ journal and benefit from:}

- Convenient online submission

- Rigorous peer review

- Open access: articles freely available online

- High visibility within the field

- Retaining the copyright to your article

Submit your next manuscript at $\boldsymbol{\nabla}$ springeropen.com 